Acta Cryst. (2002). A58 (Supplement), C9

HIGH THROUGHPUT SCREENING TECHNIQUES FOR PREFORMULATION: SALT SELECTION AND POLYMORPH STUDIES

P. Desrosiers E. Carlson W. Chandler H. Chau P. Cong R. Doolen C. Freitag S. Lin C. Masui E. Wu T. Crevier D. Mullins L. Song R. Lou J. Zhan A. Tangkilisan Q. Ung K. Phan

Symyx Technologies 3100 Central Expy SANTA CLARA CA 95051 USA

Pre-formulation is a key step in the drug development process and consists of salt selection and polymorph studies. Currently a time consuming and expensive process, there is a tremendous need to expedite these studies. Symyx Technologies has recently developed a high throughput workflow that allows both salt selection and polymorph studies to be executed in a fraction of the time using much smaller amounts of material than conventional studies. One of the major components of the integrated hardware and software system that is currently being manufactured for sale as the Symyx Polymorph Discovery ToolTM, is a parallel Crystallization StationTM. Capable of performing cooling, evaporation, and precipitation crystallizations in a fully automated manner, this crystallization station has several unique design features that allow for the hot filtration and daughtering of saturated solutions and the isolation crystals on a Universal SubstrateTM. Crystalline samples isolated in this format, are analyzed using rapid serial birefringence, Raman, XRD, and parallel melting point measurements. Using a fully functional prototype and working in collaboration with several of the leading pharmaceutical companies in the U.S., we have performed over 10,000 crystallizations of pharmaceutically active compounds in three months. Currently performing 300 crystallizations per day this system makes it possible to complete typical salt selection or polymorph studies in as little as two to three weeks.

Keywords: POLYMORPHS, SALT SELECTION, HIGH THROUGHPUT CRYSTALLIZATION

Acta Cryst. (2002). A58 (Supplement), C9

\section{CRYSTALLIZATION IN CAPILLARY SPACES: ADVANTAGES TO AUTOMATED SOLID FORM SELECTION}

G.P. Stahly L.J. Chyall B.C. Stahly D.A. Coates

SSCI Inc. 3065 Kent Avenue WEST LAFAYETTE IN 47906 USA

Crystallization from solution in capillary spaces allows high supersaturation to be attained in a quiescent environment and permits analysis of solid samples in situ by several techniques, including x-ray powder diffraction (XRPD), single crystal diffraction, Raman spectroscopy, optical microscopy, and differential scanning calorimetry. New polymorphic forms of several well-studied commercial drugs have been found in capillaries. Automated features include solubility determination, solvent handling, collection of XRPD patterns, and analysis and matching of the XRPD patterns using specially designed software. This capillary system is ideal for salt selection investigations and the solventbased portions of comprehensive polymorph screening. Other specialized screening techniques including laser nucleation and non-solvent methods will be discussed.

Keywords: AUTOMATED POLYMORPH SCREENING, AUTOMATED SALT SELECTION, CRYSTALLIZATION
Acta Cryst. (2002). A58 (Supplement), C9

HIGH-THROUGHPUT SCREENING IN SOLID FORM SELECTION A. van Langevelde E. Blomsma

Crystallics BV, Zekeringstraat 29, 1014 BV Amsterdam, The Netherlands

Active pharmaceutical ingredients (API) are often marketed in a formulated solid form, which has to meet stringent criteria on stability, aqueous solubility, toxicity etc. It is a complex issue to select the most appropriate form to market since in most cases various polymorphs exist of the free species and its different salts. To select the most appropriate form the total solid-state behavior (polymorphs and salts) has to be explored. However, crystallization of the various forms depends on many parameters, such as solvent, concentration, temperature etc., demanding a huge amount of experiments. Using highthroughput (HT) screening techniques numerous experiments are performed in parallel, accelerating the form-selection process significantly. Besides crystallization experiments in multi-well plates also automated analyses and data interpretation are key to secure a high throughput. For this purpose, an HT $\mathrm{X}$-ray powder diffraction machine giving an output of one XRPD pattern per 2 or 3 minutes is used. The XRPD patterns are automatically classified using multivariate analyses techniques. Finally, the classified forms are mapped to their crystallization conditions. Various case studies will be discussed to illustrate the solid-form screening process.

\section{Keywords: HIGH THROUGHPUT SCREENING POLYMORPHISM CRYSTALLIZATION}

Acta Cryst. (2002). A58 (Supplement), C9

\section{LOW-DENSITY ELIMINATION METHOD APPLIED TO} QUASICRYSTALS

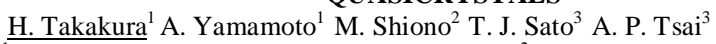

${ }^{1}$ Advanced Materials Laboratory, NIMS ${ }^{2}$ Faculty of Science, Kyushu University ${ }^{3}$ Materials Engineering Laboratory, NIMS

A novel density modification method is applied to phase reconstruction of $\mathrm{x}$ ray single crystal data of quasicrystals. This is based on the low electron density elimination (LDE) method, which is an ab initio structure determination method for crystals in the real space. The location, size and shape of the occupation domains in the n-dimensional $(\mathrm{nD})$ unit cell are obtained without any model structure. Therefore, the LDE method can be a substitute for the direct method in the quasicrystals, which is regarded as a $\mathrm{nD}$ crystal. The structure solution can easily be found in the trial sets (normally 100 sets) and is confirmed by the subsequent analysis. The phase-reconstructed structure is used as a starting model for constructing a detailed structure model of quasicrystals. The solutions of several quasicrystals (i-Al-Pd-Mn, i-Zn-MgHo and i-Cd-Yb) including a model quasicrystal (i-Al-Pd-Mn) are exemplified. The reliability and limitations of the algorithm to retrieve the quasicrystal structures will also be discussed.

Keywords: QUASICRYSTALS STRUCTURE SOLUTION, LOW DENSITY ELIMINATION 